\title{
Thriposha supplementation in healthy Sri Lankan preschool children: effect on growth
}

\author{
Hettiarachchi Manjula, Liyanage Chandrani \\ Nuclear Medicine Unit, Faculty of Medicine, Galle, Sri Lanka \\ Correspondence to: Dr. Manjula Hettiarachchi, Nuclear Medicine Unit, Faculty of Medicine, P.O. Box 70, \\ Galle,nmu_galle@yahoo.com
}

\begin{abstract}
The island-wide comprehensive supplementary feeding scheme known as 'Thriposha' programme has been in existence in the country for over three decades. Its effectiveness on growth, however, has not yet been evaluated.

Objective: We examined the effects of Thriposha dietary supplementation on anthropometric parameters (weight \& height) among preschool children in Galle.

Methods: Preschool children (aged 3-5 years) from two well-baby clinics grouped into interventional ( $\mathrm{n}=137)$ arm and control $(\mathrm{n}=130)$ arm, were fed with ordinary Thriposha $(50 \mathrm{~g} /$ day $)$ and Thriposha made without mineral and vitamin premix, respectively for a period of nine months. Height and weight were recorded using a portable stadiometer and a beam balance, respectively at baseline and after 5 months and 9 months of intervention.

Results: At the end of 9 months, weight gain in the intervention group was $+1.35(0.6) \mathrm{Kg}$, whereas it was +1.22 $(0.5) \mathrm{Kg}(\mathrm{p}=0.08$ for the between group difference) in the control. The height gain was $+4.76(1.3) \mathrm{cm}$ in intervention group, whereas control group had +3.14 (2.1) $\mathrm{cm}$ of height gain $(\mathrm{p}<0.001$ for the between group difference). There was no treatment effect on weight in children aged $<48$ months, whereas those who were gain $>48$ months in the Intervention group had significant $(\mathrm{p}=0.05)$ weight over the control group children.
\end{abstract}

Conclusions: We confirm that regular consumption of conventional Thriposha for 9 months have a positive effect on height and weight gain among preschool children.

Key words: Preschool children, dietary supplementation, weight, height.

\section{Introduction}

Child undernutrition, as indicated by linear growth failure and wasting, remains a major public health concern worldwide because of its contribution to childhood morbidity/mortality and long-term consequences, such as reduced adult muscle mass and increased obstetric risk (1). Although substantial progress has been made over the past few decades, stunting and wasting still affect about $13.5 \%$ and $14.0 \%$ preschool children, respectively. Furthermore one third of preschool children are underweight in Sri Lanka (2). Growth retardation usually begins inutero and continues during the first $2-3$ y of life as a result of inadequate food intake and infections such as diarrhoea (3). Although research on the causes of growth failure over the last half century focused initially on protein and then energy intake, more attention has been paid to micronutrients, recently.

Nutrition intervention, with a view to satisfying the minimum nutritional requirements of every citizen, has been one of the central components of Sri Lanka's welfare system since independence. Accordingly, the Government of Sri Lanka launched an island-wide comprehensive supplementary feeding scheme, known as the 'Thriposha' programme in 1973 with donor assistance, to combat the high prevalence of childhood malnutrition, low birth weight, and iron deficiency anemia in the island. Although this has been in existence for over three decades the efficacy of Thriposha in improving nutritional status in preschool children has not been evaluated so far. We therefore undertook an 
interventional trial in Southern Sri Lanka to determine the efficacy of Thriposha supplement in relation to growth, micronutrient status, bone health and cognitive abilities. This brief report addresses the effectiveness of Thriposha on linear growth of preschool children.

\section{Methods}

The study protocol for the intervention received approval from the Ethical Review Committee of the Faculty of Medicine, University of Ruhuna in 2007 and the intervention study was registered in the Sri Lanka Clinical Trial Registry (SLCTR/2007/006) managed by Sri Lanka Medical Association. The subject recruitment and experimental protocol was described in detail previously (4). Briefly, 257 healthy children aged between 3 and 5 years were randomly selected from two out of four public health clinics of University field Training Area. In order to facilitate regular monitoring over the study period without incurring huge transportation costs, 2 out of 4 public health clinics of University field Training Area (Bope-Poddala Health Division) were randomly selected. The selected two clinics were situated 10 kilometers apart and each clinic had three public health midwives (PHM) to serve the area. Then a list of children aged 3-5 years was obtained from the respective PHM and using a random number table (5), subjects were selected for the study. Next, parents of these subjects were invited to a meeting at which the purpose of the study, risks, and benefits were explained in detail. Informed consent was obtained from those families who agreed to participate.

Children who had known chronic medical conditions, took medications, or were consuming vitamin or mineral preparations preceding one month were not included in the study. Two weeks prior to the initiation of the study, each child was given $100 \mathrm{mg}$ of mebendazole daily for three days to eliminate parasites. The children were subjected to a brief physical examination. Height and weight were recorded using a portable stadiometer and a beam balance with non detachable weights respectively.

The study sample was grouped into two (interventional and control) depending on the location, after matching their initial age and anthropometry to make sure that both groups had similar characteristics at the baseline. In order to make sure that the subjects will get the recommended daily portion $(50 \mathrm{~g})$ we provided Thriposha to all children below 10 years of age in the same household. The children in the interventional group were given conventional Thriposha while the control group was fed with Thriposha made without mineral and vitamin premix (Corn-Soya-Blend- CSB) for a period of nine months. Thriposha was given to each child daily preferably two hours after either breakfast or lunch. Conventional Thriposha and CSB were ordered separately from the manufacturing plant in Kapuwatta, Ja-Ela and there were no differences in the packing material or size of the packet. Family members were blinded to the content of the packets. Distribution of Thriposha was done at monthly intervals and was handled by the research team at the respective clinic. Compliance and consumption of this meal were checked periodically, with the help of public health midwife of the relevant clinic. Further, each family unit was asked to submit remaining portion of their Thriposha in order to get a new batch and to avoid excessive consumption. Weight and height of each child were repeated after 5 months of supplementation and then after one week of completion of nine months intervention. Same instruments and same individuals were assigned to make these measurements in all three stages.

\section{Statistical analysis}

The 1978 NCHS/WHO growth reference curves were used from Epi-info version 3.0 (2003) to generate $\mathrm{Z}$ scores of weight-for-age (WAZ underweight indicator), height-for-age (HAZ stunting indicator) and weight for height (WHZ thinness). $\mathrm{Z}$ score of -2.0 was defined as the cut-off value for above indicators. Differences between groups in anthropometric indices at baseline, interim (=5 months) and final stages in the supplementation trial were tested using multivariate analysis of variance (MANOVA) repeated-measures design with supplement type as the between-subject factor (two groups) and treatment effect (baseline compared with interim/ final) as the within-subject factor. Baseline values for WAZ and HAZ were also included in the analysis as between-subject factors to correct for their possible confounding influence due to their differences at the baseline. Statistical analysis was done using open source statistical software. Descriptive statistics are expressed as the 
mean (SD). P-values less than 0.05 were considered to be significant.

\section{Results}

There were 137 subjects in the intervention group and 120 in the control group at the baseline. At the time of interim assessment at 5 months of supplementation, $8.0 \%(\mathrm{n}=11)$ children from the intervention group and $11 \%(n=14)$ children from the control group had dropped out. At the end of 9 months of intervention another 12 children had dropped out from the intervention group resulting overall drop-out rate of $17.0 \%(n=23)$. In the control group, 13 more children dropped out giving total drop-out rate of $23.0 \%(n=27)$. As such a total of 114 children in the intervention group and 93 children in the control group completed the trial. Of the 114 subjects in the intervention group 58 were males. Control group had 44 males by the completion of the trial. The mean age at the study entry was 46.5 (9.0) months and 48.3 (8.0) months for the intervention and control groups, respectively $(\mathrm{p}=0.34)$.

The means (SD) of anthropometric measurements are presented in table 1. The intervention group had a mean weight of 13.66 (2.0) $\mathrm{Kg}$ whereas control group had $13.63(2.10) \mathrm{Kg}$ at the baseline with no significant difference $(p=0.85)$ at the study entry. After 5 months of intervention mean weights were 14.42 (2.20) $\mathrm{Kg}$ and 14.31 (2.1) $\mathrm{Kg}$ in intervention and control groups, respectively $(\mathrm{p}=0.65)$. On completion, the intervention group had mean weight of 15.02 (2.30) Kg whereas mean weight of the control group was $14.84(2.1) \mathrm{Kg}(\mathrm{p}=0.72)$. Similarly there was no significant difference in either group in the change of height $(\mathrm{p}=0.65)$ or BMI $(p=0.19)$. Children in both groups had significant within group gain in weight $(\mathrm{r}=0.97, \mathrm{p}<0.001)$, height $(\mathrm{r}=0.95, \mathrm{p}<0.001)$, and BMI $(\mathrm{r}=0.80$, $\mathrm{p}<0.001)$. After correcting for significant baseline differences in WAZ and HAZ, none of those scores were found significant at the end of the study (Table 1).

Table 1: Effect of supplementation on anthropometry ${ }^{1}$

\begin{tabular}{llllll}
\hline & & n & Intervention & n & Control \\
\hline Weight $(\mathrm{Kg})$ & baseline & 137 & $13.66(2.00)$ & 120 & $13.63(2.10)$ \\
& interim & 126 & $14.42(2.20)$ & 106 & $14.31(2.10)$ \\
& final & 114 & $15.02(2.30)$ & 93 & $14.84(2.10)$ \\
Height $(\mathrm{cm})$ & baseline & 137 & $99.60(6.0)$ & 120 & $100.10(6.0)$ \\
& interim & 126 & $102.00(6.0)$ & 106 & $101.60(6.0)$ \\
& final & 114 & $104.40(6.0)$ & 93 & $103.20(6.0)$ \\
BMI $\left(\mathrm{Kg} / \mathrm{m}^{2}\right)$ & baseline & 137 & $13.72(1.0)$ & 120 & $13.54(1.0)$ \\
& interim & 126 & $13.80(1.1)$ & 106 & $13.54(1.0)$ \\
& final & 114 & $13.73(1.1)$ & 93 & $13.88(1.1)$ \\
WAZ & baseline & 137 & $-1.32(0.8)$ & 120 & $-1.60(0.9)$ \\
& interim & 126 & $-1.30(0.8)$ & 106 & $-1.60(1.0)$ \\
final & 114 & $-1.20(0.9)$ & 93 & $-1.50(0.9)$ \\
& baseline & 137 & $-0.22(0.8)$ & 120 & $-0.68(0.9)$ \\
& interim & 126 & $-0.23(0.8)$ & 106 & $-0.74(0.9)$ \\
WHZ & final & 114 & $-0.33(0.9)$ & 93 & $-0.70(0.9)$ \\
& baseline & 137 & $-1.40(0.8)$ & 120 & $-1.50(0.8)$ \\
& interim & 126 & $-1.40(0.8)$ & 106 & $-1.45(0.8)$ \\
& final & 114 & $-1.30(0.8)$ & 93 & $-1.40(0.8)$
\end{tabular}

${ }^{1}$ Results presented as mean (SD); abbreviations used, BMI - body mass index, WAZ- weight-for-age z score, HAZ - height-for-age $\mathrm{z}$ score, WHZ - weight-for-height z score

${ }^{2}$ There were significant differences $(\mathrm{p}=0.02)$ at baseline

${ }^{3}$ there were significant differences $(\mathrm{p}<0.001)$ at baseline 
The prevalence of stunting $(<-2.00$ of HAZ) was only $2 \%(n=6)$ at the study entry ( 3 subjects in each group). Twenty one percent of subjects $(\mathrm{n}=54)$ were underweight $(<-2.00 \mathrm{WAZ}) ; 18 \%(\mathrm{n}=24)$ in the intervention and $25 \%(\mathrm{n}=30)$ in the control groups, respectively. The overall prevalence of thinness $(<-2.00 \mathrm{WHZ})$ was $16 \%(\mathrm{n}=41) ; 15 \%(\mathrm{n}=20)$ in the interventional group and $18 \%(\mathrm{n}=21)$ in the control group. By the end of intervention none of the subjects were found stunted. Prevalence of underweight dropped to $13 \%(\mathrm{n}=18)$ in the intervention group $(\mathrm{p}=0.05)$ whereas $23 \%(\mathrm{n}=28)$ of children remained underweight in the control group. Prevalence of thinness remained unchanged; $13 \%(\mathrm{n}=18)$ and $16 \%$ $(\mathrm{n}=19)$ in the intervention and control groups, respectively.

Figure demonstrates the mean gains in weight and height of subjects who consumed Thriposha (either conventional or CSB) during the study period. The total effect on weight gain in the intervention group was $+1.35(0.6) \mathrm{Kg}$, whereas it was $+1.22(0.5) \mathrm{Kg}$ $\mathrm{p}=0.08)$ in the control. With the improved height gain, children in the intervention group showed a drop in BMI gain.
With evidence of positive effect on height gain we subdivided the children into two categories according to their age at study entry; younger group; aged $<48$ months $(\mathrm{n}=73$ in the intervention and $\mathrm{n}=43$ in the control groups) and older group; aged $>48$ months $(\mathrm{n}=41$ and 51 in the intervention and control groups, respectively) and analyzed the effect of intervention on weight and height. Results are summarized in table 2. Younger age category of the intervention group did not have a significant change in weight but, they had higher height at the end of nine months (101.24 vs. $99.31 \mathrm{~cm}, \mathrm{p}=0.02)$ in the control group resulting significant treatment effect after the intervention $(p=0.01)$. Older children of the intervention group showed a similar effect on weight at the final assessment (Table 2). However, they had a significant improvement $(\mathrm{p}=0.01)$ in height even at the interim assessment conducted after 5 months of study entry.

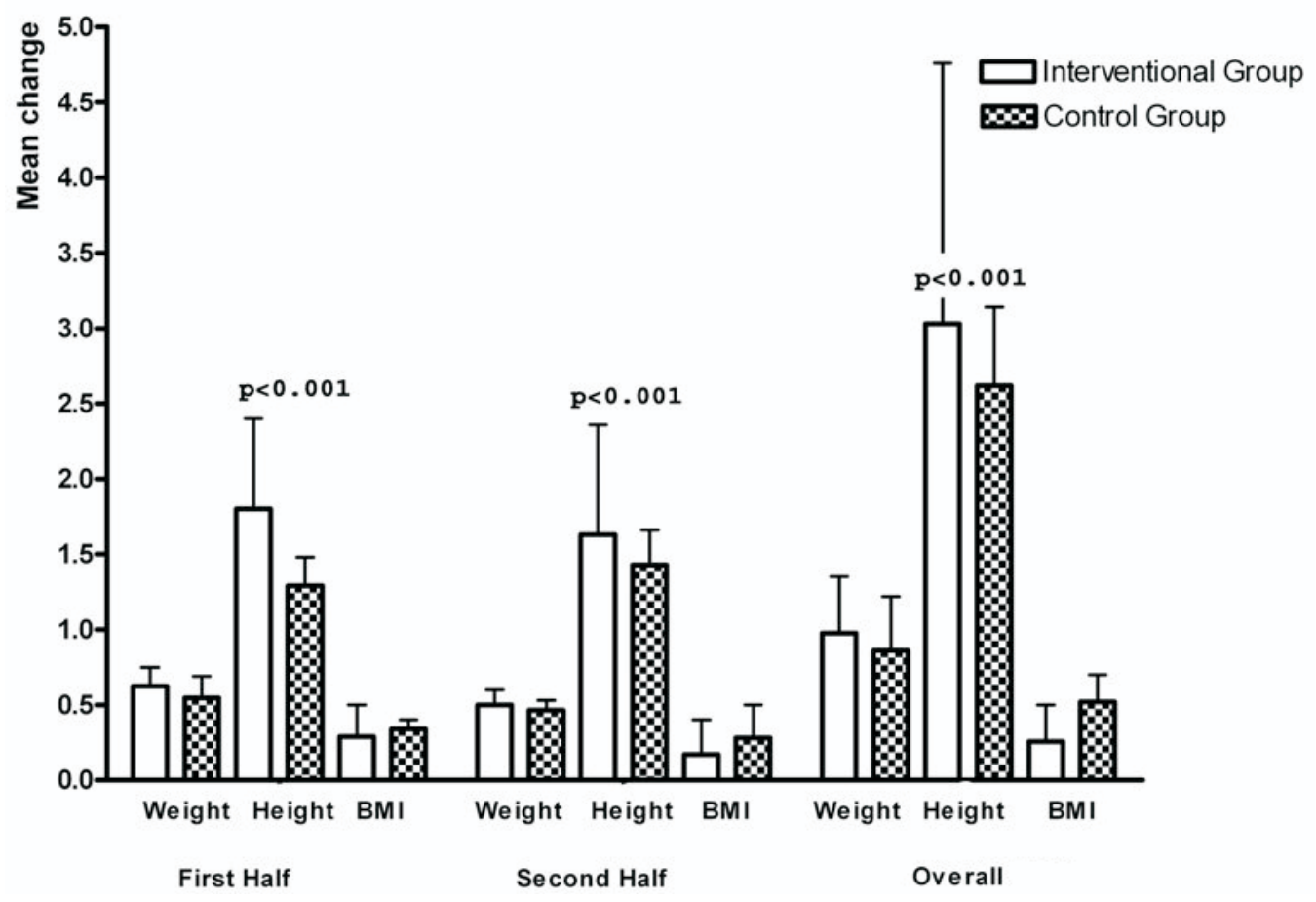

Figure: Mean change in anthropometric parameters during the intervention ${ }^{1}$

\footnotetext{
${ }^{1}$ Results presented in mean (+SEM); abbreviations used, BMI - body mass index; First half -difference between baseline and interim assessment; Second half - difference between interim and final assessment; Over all - difference between baseline and final assessment; $p$-values derived from multivariate analysis of variance (repeated measures design).
} 
Table 2: Effect of supplementation on anthropometry at different age groups ${ }^{1}$

\begin{tabular}{|c|c|c|c|c|c|}
\hline & & Interventional & Control & p-value ${ }^{2}$ & p-value ${ }^{3}$ \\
\hline \multirow[t]{2}{*}{ Age $3 \& 4$ years } & (<48 months) & & & & \\
\hline & $\mathrm{n}$ & 73 & 42 & & \\
\hline \multirow[t]{3}{*}{ Weight (Kg) } & baseline & $12.77(1.4)$ & $12.41(1.7)$ & 0.22 & \\
\hline & interim & $13.48(1.5)$ & $13.10(1.7)$ & 0.22 & \\
\hline & final & $14.06(1.5)$ & $13.64(1.8)$ & 0.18 & 0.20 \\
\hline \multirow[t]{3}{*}{ Height $(\mathrm{cm})$} & baseline & 96.64 (4.6) & $95.93(5.0)$ & 0.44 & \\
\hline & Interim & $98.92(4.2)$ & $97.44(4.9)$ & 0.09 & \\
\hline & final & $101.24(4.0)$ & $99.31(4.8)$ & 0.02 & 0.01 \\
\hline
\end{tabular}

Age 5 years ( $>48$ months)

\begin{tabular}{|c|c|c|c|c|c|}
\hline & $\mathrm{n}$ & 41 & 51 & & \\
\hline \multirow{3}{*}{ Weight (Kg) } & baseline & $15.25(1.9)$ & $14.63(1.8)$ & 0.11 & \\
\hline & interim & $16.08(2.2)$ & $15.31(1.8)$ & 0.07 & \\
\hline & final & $16.74(2.4)$ & $15.83(1.8)$ & 0.05 & 0.05 \\
\hline \multirow[t]{3}{*}{ Height $(\mathrm{cm})$} & baseline & $104.89(4.2)$ & $103.49(4.4)$ & 0.13 & \\
\hline & Interim & $107.48(4.8)$ & $104.95(4.6)$ & 0.01 & \\
\hline & final & $109.90(4.9)$ & $106.44(4.7)$ & $<0.001$ & 0.01 \\
\hline
\end{tabular}

${ }^{1}$ Results presented as mean (SD)

${ }^{2}$ Students t-test comparing interventional and control groups

${ }^{3}$ Repeated measures multivariate analysis of variance

\section{Discussion}

This paper describes the impact of daily supplementation of ready-to-eat cereal-based meal for a period of 9 months among preschool children on height and weight gain. A significant gain in height was seen but the impact on weight was limited to specific age group. This intervention was controlled with the provision of CSB, and as such, the intake of energy and protein was controlled throughout the intervention. The observed differences in growth therefore can be attributed to the micronutrient content of the supplement. The micronutrients associated with linear growth are those believed to be nutritionally significant and reduced availability of these micronutrients below a certain threshold consistently leads to a reduction in one or more physiologically important functions. These micronutrients have been defined by a WHO Expert Committee to include iodine, zinc, selenium, copper, molybdenum, chromium, vitamin A and calcium (6). Multiple nutrient deficiencies are likely to occur in children in developing countries and supplementation with individual micronutrients can be expected to have only a limited impact on growth.

Studies in various settings have shown that deficiencies of iron, vitamin $\mathrm{A}$, zinc and other micronutrients often coexist $(7,8)$. However, most of the studies have focused on the role of one or two micronutrient deficiencies on an individual linear growth faltering and the extent to which supplementation of such alone in improving growth (9). Ramakrishnan et al., (1) have noted that vitamin A or iron administered as single nutrients had no significant effect on growth, suggesting that correcting multiple nutrient deficiencies simultaneously is more efficacious. The increased effectiveness of multi mineral-vitamin interventions when compared with either one or two mineral interventions may be explained by the high prevalence of concurrent micronutrient deficiencies 
in these settings $(8,10)$ and/or the positive synergistic effects between some of these nutrients at the level of both absorption and metabolism (for example, vitamin $\mathrm{A}$ and iron, vitamin $\mathrm{A}$ and zinc, iron and vitamin C) (10). Brown et al., (11) suggest that the effectiveness of the multimicronutrient interventions in improving growth may have been due to zinc, which was included in all interventions. Further, our results also suggest that recent concerns about the competitive interaction between iron and zinc (12) may not be a problem, at least for growth outcomes, when zinc is included in a multivitamin-mineral supplement.

There are several possible explanations for the observed results. First, all subjects in the study were given the project foods free of charge, which may have improved overall food availability for them. It would have been desirable for study purposes to include a group assigned with normal diet alone, but this was not considered acceptable because of the known nutritional limitations. Second, the attention received by children through regular weekly visits may have influenced the mothers' childcare and feeding practices, and the regular morbidity surveillance may have prompted them to seek treatment earlier for their sick kids (13). Third, differences in the timing of data collection for the longitudinal compared with the cross sectional studies could have confounded the results, even though this was controlled for during data analysis. However, this would most likely have worked against finding greater growth in the older children because their hungry season was greater with kindergarten schooling than in the younger children group. Finally, the precautions taken to reduce contamination of project foods by providing Thriposha every 8 weeks to all mothers may have reduced the infection rates, which has been negatively associated with growth in other studies (14). In hindsight, the study design would have been stronger had we included in the randomized longitudinal study another group that was not provided with any of the project foods, but for budgetary and practical reasons this was not considered feasible or acceptable to the community. All of the above explanations may account for the improvement in growth of the intervention as well as control groups and the lack of significant differences in growth between groups.
The improved growth shown in the intervention group was not due to a higher energy intake from the cereal based meal itself because both groups got the same amount of additional energy from the food supplement ( $419 \mathrm{~kJ} /$ child) daily. But there are other potential explanations. One of the cardinal features of zinc deficiency is growth failure; thus, it is possible that the increase in zinc intake facilitated an augmented lean tissue accretion or increased utilization of the energy provided. Such a response was described in zinc-supplemented Ugandan preschool children (15). The concept of "hidden hunger" associated with micronutrient deficiency has been described in recent years (16), and this may provide an alternative explanation for the improvement in growth. Lawless et al., (17) reported improvements in growth and appetite as a result of iron supplementation on the basis of the results of an appetite test and questionnaires. It is therefore possible that the intervention group may have had higher energy intake as a result of increased appetite due to improvements in their folate and iron status.

We consider the randomization done in the present study was effective because the anthropometric indices at baseline, most of the measurements made during the study period, and other characteristics of the children and their families at baseline were similar between the two groups. Our results also indicate that growth was stimulated in the older children after 5 months of supplementation and continuation of supplementation yielded ongoing positive effects on growth. Effect on height was evident even at $5^{\text {th }}$ month of intervention. Additional studies are needed to measure growth responses when supplementation is begun at younger ages and continued for a longer time. A low interaction between treatment and the degree of stunting appeared in the study could be due to the low degree of stunting in the group studied which was not severe enough to show any treatment effect.

In conclusion, we document a marginal positive effect of Thriposha supplementation on the weight gain in children older than 4 years with a significant positive effect on height gain among preschool children. Significant heterogeneity was evident, and its predictors depend on age and the duration of supplementation. However, these predictors of heterogeneity should be viewed only as exploratory and requiring confirmation, not as conclusive. The results of our study show that micronutrients are a 
limiting factor for the growth of young preschool children. We confirm that multi-micronutrient interventions that provide the recommended daily allowances of iron, zinc, vitamin A, folic acid, and B vitamins have a positive effect on height and weight gain in children. The public health interventions aimed at improving the nutritional status and growth should consider improvements in micronutrient intakes as an essential component.

\section{Acknowledgements}

This project received funding from the International Atomic Energy Agency research grant IAEA-SRL11958. Conventional Thriposha and the CSB were received freely through Thriposha programme coordinator of the Ministry of Health, Sri Lanka. Managerial and technical staff of the Thriposha manufacturing plant assured continuous supply of supplement throughout the study. We thank the staff of the Nuclear Medicine Unit for helping in anthropometric measurements of the children. The parents of the study subjects are acknowledged for their cooperation in meal preparation.

\section{References}

1. Ramakrishnan U., McCabe G. \& Martorell R. Multimicronutrient interventions but not vitamin A or iron interventions alone improve child growth: Results of 3 meta-analyses. Journal of Nutrition, 2004; 134; 2592-2602.

2. Nutritional Status of Pre-School Children (2009) Department of Census and Statistics - Sri Lanka.

3. Schroeder D.G. Malnutrition. In: Nutrition and Health in Developing Countries (Eds. Semba R.D. \& Bloem M.W.), Humana Press, Totowa, NJ. 2001: pp. 393-426.

4. Hettiarachchi M. \& Liyanage C. Efficacy of Thriposha supplementation in improving the micronutrient status of Sri Lankan preschool children. Ceylon Medical Journal. 2010 Accepted for publication.

5. Anderson A.J.B. Interpreting Data: A first course in statistics. $8^{\text {th }}$ edition. Chapman and Hall, UK. 1989.

6. World Health Organization. Trace elements in human nutrition and health. WHO, Geneva. 1996.

7. Rosado J.L. Separate and joint effects of micronutrient deficiencies on linear growth. Journal of Nutrition, 1999; 129: 531S-533S.
8. Hettiarachchi M., Liyanage C., Wickremasinghe R., Hillmers D. \& Abrams S. Prevalence and severity of micronutrient deficiency: a cross-sectional study among adolescents in Sri Lanka Asia Pacific Journal of Clinical Nutrition, 2006; 15(1): 56-63.

9. Bhandari N., Bahl R. \& Taneja S. (2001) Effect of micronutrient supplementation on linear growth of children. British Journal of Nutrition, 85(2); 131S-137S.

10. Dijkhuizen M.A., Wieringa F.T., West C.E. \& Muhilal. Micronutrient deficiency and supplementation in Indonesian infants. Interactions between micronutrients. Advances in Experimental and Medical Biology. 2003; 531: 359-368.

11. Brown K.H., Peerson J.M., Rivera J. \& Allen L.H. Effect of supplemental zinc on the growth and serum zinc concentrations of prepubertal children: a meta-analysis of randomized controlled trials. American Journal of Clinical Nutrition, 2002; 75: 1062-1071.

12. Zlotkin S., Arthur P., Schauer C., Antwi K.Y., Yeung G. \& Piekarz A. Home fortification with iron and zinc sprinkles or iron sprinkles alone successfully treats anemia in infants and young children. Journal of Nutrition, 2003; 133: 1075-1080.

13. Vijayaraghavan K., Radhaiah G. \& Reddy V. Vitamin A supplementation and childhood mortality. Lancet, 1992; 340:1358-1359.

14. Becker S., Black R.E. \& Brown K.H. Relative effects of diarrhea, fever and dietary energy intake on weight gain in rural Bangladeshi children. American Journal of Clinical Nutrition.1991; 53:1499-1503.

15. Kikafunda J.K., Walker A.F., Allan E.F. \& Tunwine J.K. Effect of zinc supplementation on growth and body composition of Ugandan preschool children: a randomized, controlled, intervention trial. American Journal of Clinical Nutrition, 1998; 68: 1261-1266.

16. Maberly G.F., Trowbridge F.L., Yip R., Sullivan K.M. \& West C.E. Programs against micronutrient malnutrition: ending hidden hunger. Annual Review in Public Health, 1994; 15: 277-301.

17. Lawless J.W., Latham M.C., Stephenson L.S., Kinoti S.N. \& Pertet A.M. Iron supplementation improves appetite and growth in anemic Kenyan primary school children. Journal of Nutrition, 1994; 124: 645-654. 